\title{
Empirical Modeling of Average Cutting Speed during WEDM of Hastelloy C22
}

\author{
Bhupinder Singh ${ }^{1}$, and Joy Prakash Misra ${ }^{1}$ \\ ${ }^{1}$ Dept. of Mechanical Engineering, National Institute of Technology, Kurukshetra, India
}

\begin{abstract}
Cutting speed (CS) is a key performance measure to achieve optimal utilization of the WEDM process. However, input process parameters of WEDM and combination of wire and workpiece material greatly hamper CS and hence productivity and machining efficiency. Therefore, it is essential to pick the right combination of parameters and wire and workpiece material to obtain better CS. In this paper, four process parameters: Pulse-on time, Pulse-off time, Spark-gap voltage, and Peak current were chosen to develop an empirical model for CS during WEDM of Hastelloy C22 to provide a guideline to the potential users of the technique. This paper describes the response surface methodology (RSM) based mathematical modeling for average cutting speed. Furthermore, analysis of variance (ANOVA) was applied to find out significant process parameters and it was depicted that pulse on time and peak current were the major parameters affecting CS.
\end{abstract}

\section{Introduction}

WEDM is one of the extensively accepted nonconventional machining processes employed to manufacture components with complex shapes, profile and sharp edges that are difficult-to-machine by other traditional and non-traditional machining processes [1] and hence, this process has wide application areas including aerospace industry, medical implants, electronic industry, automobile industries, etc. It is an electro-thermal production type advanced machining process in which removal of material occurs due to melting and vaporization owing to a series of sparks between workpiece and wire electrode (thin copper, brass or tungsten of diameter $0.05-0.3 \mathrm{~mm}$ ) submerged in a dielectric fluid (deionized water). Both electrodes are connected to a pulsed DC power supply. There is a gap between the wire and the workpiece usually ranges from $0.025 \mathrm{~mm}$ to $0.050 \mathrm{~mm}$ and is continuously maintained by a computer-controlled positioning system. The wire is kept under tension by tensioning device to overcome the inaccuracies in the machined parts. The material is removed by the series of electrical discharges between wire and workpiece in the presence of deionized water. At the point, ionization occurs and electricity flows through the ionized column of dielectric fluid between the positive and negative electrode. Once ionization takes place, the dielectric becomes heated from the flow of electricity and then changes into a gas known as plasma. Under this condition electrons rapidly pass through the ionized plasma in the form of a spark. The positive ions from the workpiece attracted to the negatively charged electrode and the negatively charged electron collide with the workpiece and produce a spark, and this spark melts and vaporizes the small amount of material from the workpiece [2-4]. The details of the mechanism of material removal, process parameters, the influence of process parameters on measures of process performance are available in the literature [5-8]. In this study, an attempt has been made to develop an empirical modeling of cutting speed for WEDM of Hastelloy C22 for providing a guideline to the future users of the technique in selecting influencing process parameters for obtaining the desired outcome. Figure 1 illustrates the schematic of WEDM process.

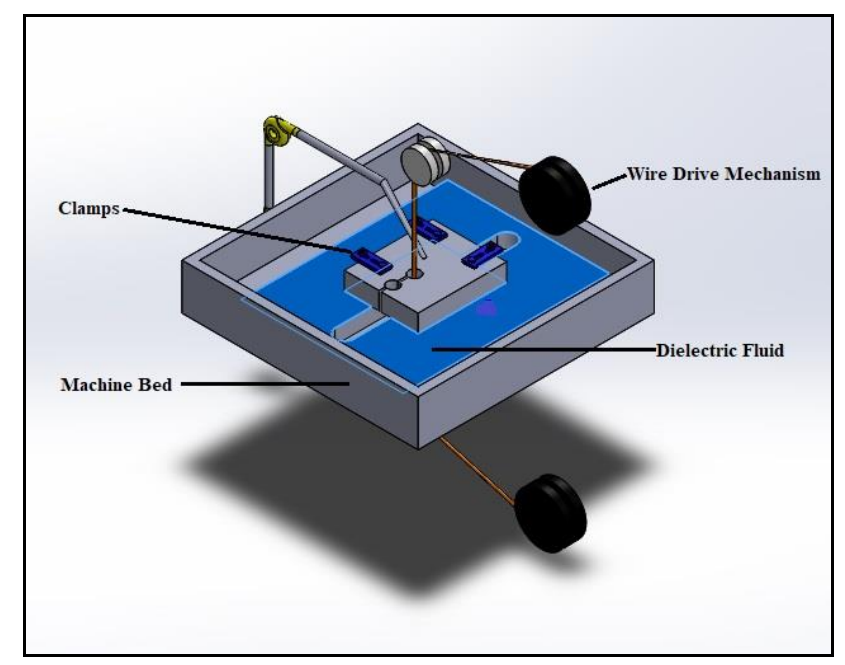

Figure 1. Schematic of WEDM process (Guitrau, 1997)

\section{Experimental procedure}




\subsection{Experimental setup and details}

The experimental investigation has been conducted on 734 sprintcut WEDM (Make: Electronica Machine Tool). This machine setup comprises of four major subcomponents: power supply system, dielectric system, positioning system, and wire drive mechanism. The detailed functions of each sub-element are available in reference [1,2]. From literature survey, it has been observed that most of the research work carried out on steel and steel alloys, aluminium alloys and titanium alloys $[6,9,10]$. Less research work has been reported on nickel-base super alloys and therefore it is very much essential to explore WEDM of nickel-base super alloys owing to its industrial importance in gas turbine and aerospace industry. Traditionally, combustor components have been made-out of hastelloy C22 sheets. So, in this research work hastelloy $\mathrm{C} 22$ has been selected as workpiece material. Rectangular Hastelloy C22 sheet of $100 \mathrm{~mm}$ (length), $100 \mathrm{~mm}$ (width), $10 \mathrm{~mm}$ (thickness) was used for the experimentation work and $5 \mathrm{~mm} \times 5 \mathrm{~mm}$ square cuts are taken on the workpiece. Half hard-brass wire ( $\phi=0.25 \mathrm{~mm}$ ) with $900 \mathrm{~N} / \mathrm{mm}^{2}$ is used as cutting tool, while de-ionized water was used as a dielectric. The process parameters of WEDM process are broadly classified into four groups: power supply related parameters: Pulse-on time, Pulse-off time, Servo voltage, Peak current, Servo feed, etc.; electrode related parameters: wire material, wire diameter, wire feed rate, wire tension, wire-offset, etc.; workpiece related parameters: workpiece height, thermal conductivity, electrical conductivity, etc.; dielectric related parameters: dielectric type, dielectric flow rate, conductivity of dielectric, etc.

\subsection{Design of experiments}

In this study an effort has been made to find out the relationship between input and response parameters by developing empirical model for CS. This experimental study consists of four factors: $T_{\text {on }}, T_{\text {off }}, S v$, Ip and each factor is having three levels. The input process parameters values and their levels for the main experiments were decided on the basis of pilot experiments. Which are conducted by using OFTA. It is obvious from the results of pilot experiments that, variation of wire feed rate, wire tension and servo feed have some insignificant effect on the CS. Therefore, these parameters were kept fixed during experimentation. The dielectric fluid temperature, conductivity, dielectric pressure and dielectric chilling medium $\left(-2^{\circ} \mathrm{C}\right)$ were also kept constant. In this study, experimental runs are designed and planned according to the Box-Behnken Design (BBD) of response surface methodology (RSM). Hence, total twenty-nine runs including five replications of centre run are required [11]. The parametric combinations for different trial runs are presented in Table 3. Table 1 and Table 2 shows the levels and values for input and fixed process parameters.
Table 1. Ranges and levels of input process parameters.

\begin{tabular}{|l|c|c|c|c|}
\hline \multicolumn{5}{|c|}{ Input process parameters } \\
\hline \multirow{2}{*}{ Parameters } & \multirow{2}{*}{ Ranges } & \multicolumn{3}{|c|}{ Levels } \\
\cline { 3 - 5 } & & L1 & L2 & L3 \\
\hline Pulse-on time $\left(\mathrm{T}_{\text {on }}\right)$ & $105-131 \mu \mathrm{s}$ & 105 & 118 & 131 \\
\hline Pulse-off time $\left(\mathrm{T}_{\text {off }}\right)$ & $30-60 \mu \mathrm{s}$ & 30 & 45 & 60 \\
\hline Peak current $(\mathrm{Ip})$ & $40-220 \mathrm{~A}$ & 40 & 130 & 220 \\
\hline Servo voltage $(\mathrm{Sv})$ & $20-80 \mathrm{~V}$ & 20 & 50 & 80 \\
\hline
\end{tabular}

Table 2. Values of fixed parameters.

\begin{tabular}{|c|c|c|}
\hline \multicolumn{3}{|c|}{ Fixed process parameters } \\
\hline Parameters & Value & Fixing criteria \\
\hline Dielectric fluid & De-ionized Water & \multirow{6}{*}{$\begin{array}{l}\text { Literature review } \\
\quad \text { and } \\
\text { Pilot experiments }\end{array}$} \\
\hline Peak Voltage & $2 \mathrm{~m} / \mathrm{c}$ unit & \\
\hline Water Pressure & $1 \mathrm{~m} / \mathrm{c}$ unit & \\
\hline Wire Feed & $8 \mathrm{~m} / \mathrm{c}$ unit & \\
\hline Wire Tension & $12 \mathrm{~m} / \mathrm{c}$ unit & \\
\hline Servo Feed & $2200 \mathrm{~m} / \mathrm{c}$ unit & \\
\hline $\begin{array}{l}\text { Workpiece } \\
\text { material }\end{array}$ & Hastelloy C22 & $\begin{array}{l}\text { Industrial } \\
\text { application }\end{array}$ \\
\hline
\end{tabular}

\section{Result and discussion}

Table 3 shows the order, parametric combination, and design of experiments based on the coded surfaces and corresponding results for cutting speed. It is evident from Table 3 that maximum value of CS of $4.49 \mathrm{~mm} / \mathrm{min}$ is obtained for experiment no. 20 ( $\mathrm{T}_{\text {on }}: 125 \mu \mathrm{s}$; $\mathrm{T}_{\text {off: }} 50 \mu \mathrm{s}$; Ip: $220 \mathrm{~A}$; and $\mathrm{Sv}$ : $50 \mathrm{~V}$ ) while experiment no. 3 ( $\mathrm{T}_{\text {on }}$ : $105 \mu \mathrm{s}$; $\mathrm{T}_{\text {off: }} 55 \mu \mathrm{s}$; Ip: $130 \mathrm{~A}$; and Sv: $50 \mathrm{~V}$ ) provides the lowest value of CS of $0.47 \mathrm{~mm} / \mathrm{min}$ and \% of change is $86.89 \%$. It is also evident that no wire breakage has been encountered in any experiment.

Table 3. Experimental design and corresponding results.

\begin{tabular}{|c|c|c|c|c|c|}
\hline \multirow{2}{*}{$\begin{array}{c}\text { Ex. } \\
\text { No. }\end{array}$} & \multicolumn{3}{|c|}{ Values of input process } & \multirow{2}{*}{$\begin{array}{c}\text { CS } \\
\text { parameters }\end{array}$} \\
\cline { 2 - 5 } & $\begin{array}{c}\mathbf{T}_{\text {on }} \\
(\boldsymbol{\mu s})\end{array}$ & $\begin{array}{c}\mathbf{T}_{\text {off }} \\
(\boldsymbol{\mu} \mathbf{\text { s }})\end{array}$ & $\begin{array}{c}\text { IP } \\
(\mathbf{A})\end{array}$ & $\begin{array}{c}\mathbf{V} \mathbf{\text { V }} \\
(\mathbf{V})\end{array}$ & \\
\hline 01 & 105 & 45 & 130 & 50 & 0.80 \\
\hline 02 & 125 & 45 & 130 & 50 & 3.85 \\
\hline 03 & 105 & 55 & 130 & 50 & $\mathbf{0 . 4 7}$ \\
\hline 04 & 125 & 55 & 130 & 50 & 2.23 \\
\hline 05 & 115 & 50 & 40 & 30 & 1.07 \\
\hline 06 & 115 & 50 & 220 & 30 & 2.85 \\
\hline 07 & 115 & 50 & 40 & 70 & 0.56 \\
\hline 08 & 115 & 50 & 220 & 70 & 1.31 \\
\hline 09 & 105 & 50 & 130 & 30 & 0.69 \\
\hline 10 & 125 & 50 & 130 & 30 & 3.28 \\
\hline 11 & 105 & 50 & 130 & 70 & 0.51 \\
\hline 12 & 125 & 50 & 130 & 70 & 1.81 \\
\hline 13 & 115 & 45 & 40 & 50 & 1.05 \\
\hline 14 & 115 & 55 & 40 & 50 & 0.61 \\
\hline 15 & 115 & 45 & 220 & 50 & 2.79 \\
\hline 16 & 115 & 55 & 220 & 50 & 1.40 \\
\hline 17 & 105 & 50 & 40 & 50 & 0.48 \\
\hline 18 & 125 & 50 & 40 & 50 & 0.88 \\
\hline 19 & 105 & 50 & 220 & 50 & 0.65 \\
\hline 20 & 125 & 50 & 220 & 50 & $\mathbf{4 . 4 9}$ \\
\hline 21 & 115 & 45 & 130 & 30 & 2.87 \\
\hline 22 & 115 & 55 & 130 & 30 & 1.61 \\
\hline 23 & 115 & 45 & 130 & 70 & 1.40 \\
\hline
\end{tabular}




\begin{tabular}{|c|c|c|c|c|c|}
\hline 24 & 115 & 55 & 130 & 70 & 0.75 \\
\hline $25(\mathrm{C})$ & 115 & 50 & 130 & 50 & 1.55 \\
\hline $26(\mathrm{C})$ & 115 & 50 & 130 & 50 & 1.54 \\
\hline $27(\mathrm{C})$ & 115 & 50 & 130 & 50 & 1.54 \\
\hline $28(\mathrm{C})$ & 115 & 50 & 130 & 50 & 1.54 \\
\hline $29(\mathrm{C})$ & 115 & 50 & 130 & 50 & 1.54 \\
\hline
\end{tabular}

Quadratic model is providing the lowest $\mathrm{p}$ value. Hence quadratic model is selected for analysis of variance for the present study. Table 4 presents mean square, the sum of square, the degree of freedom, $F$ value and $p$ value for different terms of the quadratic model. Compare the $\mathrm{p}$ value for the F-test to your significance level. If the pvalue is less than the significance level, your sample data provide sufficient evidence to conclude that your regression model fits the data better than the model with no independent variables. The model F-value of 64.41 with corresponding p-value of $<0.0001$ implies that the model is highly significant as shown in Table 4 . There is only a $0.01 \%$ chance that a "Model F-Value" this large could occur due to noise. Values of "Prob $>$ F" less than 0.05 indicate model terms are significant. In this case $T_{\text {on }}$, $\mathrm{T}_{\text {off }}, \mathrm{Ip}, \mathrm{T}_{\text {on }} \times \mathrm{Ip}, \mathrm{T}_{\text {off }} \times \mathrm{Sv}, \mathrm{Sv} \times \mathrm{Ip}, \mathrm{T}_{\text {on }}{ }^{2}, \mathrm{~T}_{\text {off }}{ }^{2}, \mathrm{~Sv}^{2}, \mathrm{Ip}^{2}$ are significant model terms. Values greater than 0.1 indicate the model terms are not significant.

Table 4. Analysis of variance results (ANOVA).

\begin{tabular}{|c|l|l|l|l|l|}
\hline Source & \multicolumn{1}{|c|}{ SS } & DF & \multicolumn{1}{|c|}{ MS } & $\begin{array}{c}\text { F- } \\
\text { Value }\end{array}$ & P> F \\
\hline Model & 30.61 & 10 & 3.06 & 64.41 & $<0.0001$ \\
\hline A-T $_{\text {on }}$ & 13.98 & 1 & 13.98 & 294.20 & $<0.0001$ \\
\hline B-T off & 2.71 & 1 & 2.71 & 56.94 & $<0.0001$ \\
\hline C-Sv & 6.51 & 1 & 6.51 & 137.05 & $<0.0001$ \\
\hline D-Ip & 3.04 & 1 & 3.04 & 63.94 & $<0.0001$ \\
\hline AB & 0.42 & 1 & 0.42 & 8.77 & 0.0084 \\
\hline AC & 2.97 & 1 & 2.97 & 62.43 & $<0.0001$ \\
\hline AD & 0.41 & 1 & 0.41 & 8.53 & 0.0091 \\
\hline BC & 0.22 & 1 & 0.22 & 4.70 & 0.0438 \\
\hline BD & 0.091 & 1 & 0.091 & 1.93 & 0.1822 \\
\hline CD & 0.27 & 1 & 0.27 & 5.61 & 0.0292 \\
\hline Residual & 0.86 & 18 & 0.048 & & \\
\hline $\begin{array}{c}\text { Lack of } \\
\text { Fit }\end{array}$ & 0.86 & 14 & 0.061 & 4443.7 & $<0.0001$ \\
\hline $\begin{array}{c}\text { Pure } \\
\text { Error }\end{array}$ & $5.5 \times$ & 4 & $1.37 \times$ & & \\
\hline Cor Total & 31.47 & 28 & & & \\
\hline
\end{tabular}

In WEDM, cutting speed changes due to the combination of wire tool and workpiece material, the polarity of the voltage to apply, the duration of the spark, discharge energy during the spark and so on. The average cutting speed value is plotted against the process inputs to investigate the effects of input process parameters on a measure of process performance. Figure 2 illustrates the effects of $T_{\text {on }}, T_{\text {off }}$, Ip and $\mathrm{Sv}$ on CS. It is evident that $T_{\text {on }}$ is most significant effect on CS. It is clear from the figure 2 that CS continuously increases by increasing $\mathrm{T}_{\text {on }}$ and decreases by decreasing $\mathrm{T}_{\text {on }}$. It has been also observed that Ip is having some similar effects on CS. On the other hand, it has been observed that $\mathrm{T}_{\text {off }}$ and $\mathrm{Sv}$ is having some adverse effects on CS. Where CS values decreases by increasing the $\mathrm{T}_{\text {off }}$ and $\mathrm{Sv}$, whereas by decreasing $\mathrm{T}_{\text {off }}$ and $\mathrm{Sv}$ some increment was noticed in CS. $\mathrm{T}_{\text {off }}$ plays a vital role in $\mathrm{CS}$, at the lower level of $\mathrm{T}_{\text {on }}$ with the increase in $\mathrm{T}_{\text {off }}$ level CS will be lower compared with the higher level of $\mathrm{T}_{\text {on }}$. Alternatively, a Smaller value of servo voltage narrows down the spark gap, which leads to a greater number of sparks per unit time. It speeds up the machining rate and thus the amount of material removal from the workpiece and tool [7].

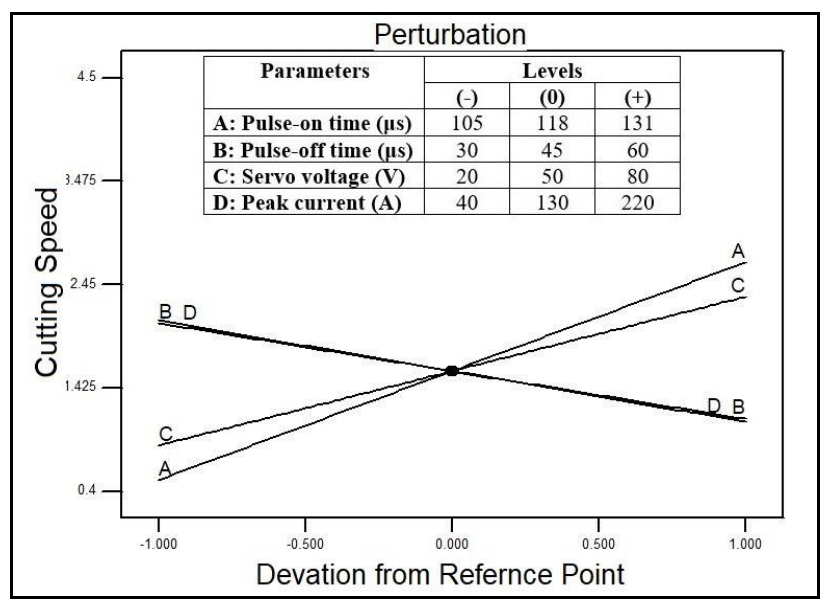

Figure 2. Effects of $\mathrm{T}_{\text {on, }} \mathrm{T}_{\text {off, }} \mathrm{Sv}$ and Ip on cutting speed [X-axis in coded value]

It is evident from analysis of variance table that the input parameters also have interaction effects on process performance characteristic. Figure 3 illustrates the interaction effects of: (a) Ip-T on vs CS; (b) $\mathrm{Sv}_{\text {- }}$ off vs CS; and (c) Ip-Sv vs CS. After that, a regression model of CS has been developed (in actual value) as illustrated using equation (1). The coefficient of determination $\left(\mathrm{R}^{2}\right)$ value of the developed model is found as 0.9728; is established the creditability of this model.

$\mathrm{CS}=+1.59+1.08 \times \mathrm{A}-0.47 \times \mathrm{B}+0.74 \times \mathrm{C}-0.50 \times \mathrm{D}$

$-0.32 \times \mathrm{A} \times \mathrm{B}+0.86 \times \mathrm{A} \times \mathrm{C}-0.32 \times \mathrm{A} \times \mathrm{D}$

$-0.24 \times \mathrm{B} \times \mathrm{C}+0.15 \times \mathrm{B} \times \mathrm{D}-0.26 \times \mathrm{C} \times \mathrm{D}$

( $\mathrm{A}=$ Pulse-on time; $\mathrm{B}=$ Pulse-off time; $\mathrm{C}=$ Peak current; $\mathrm{D}=$ Servo Voltage)

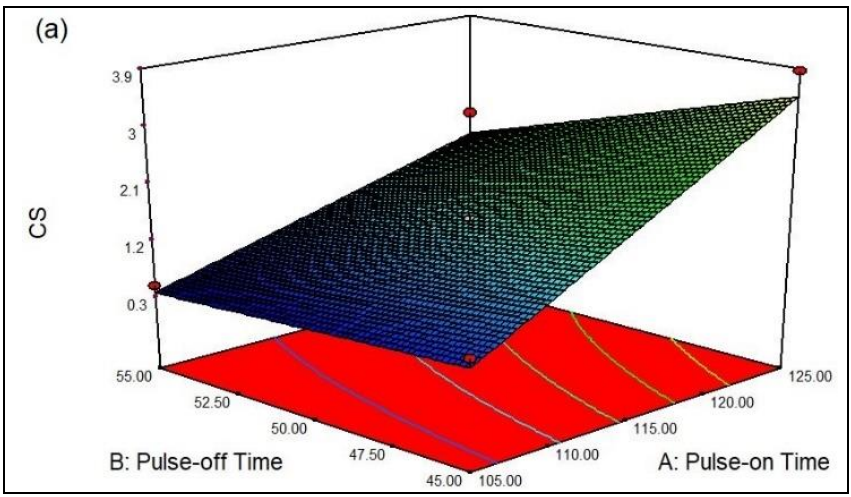



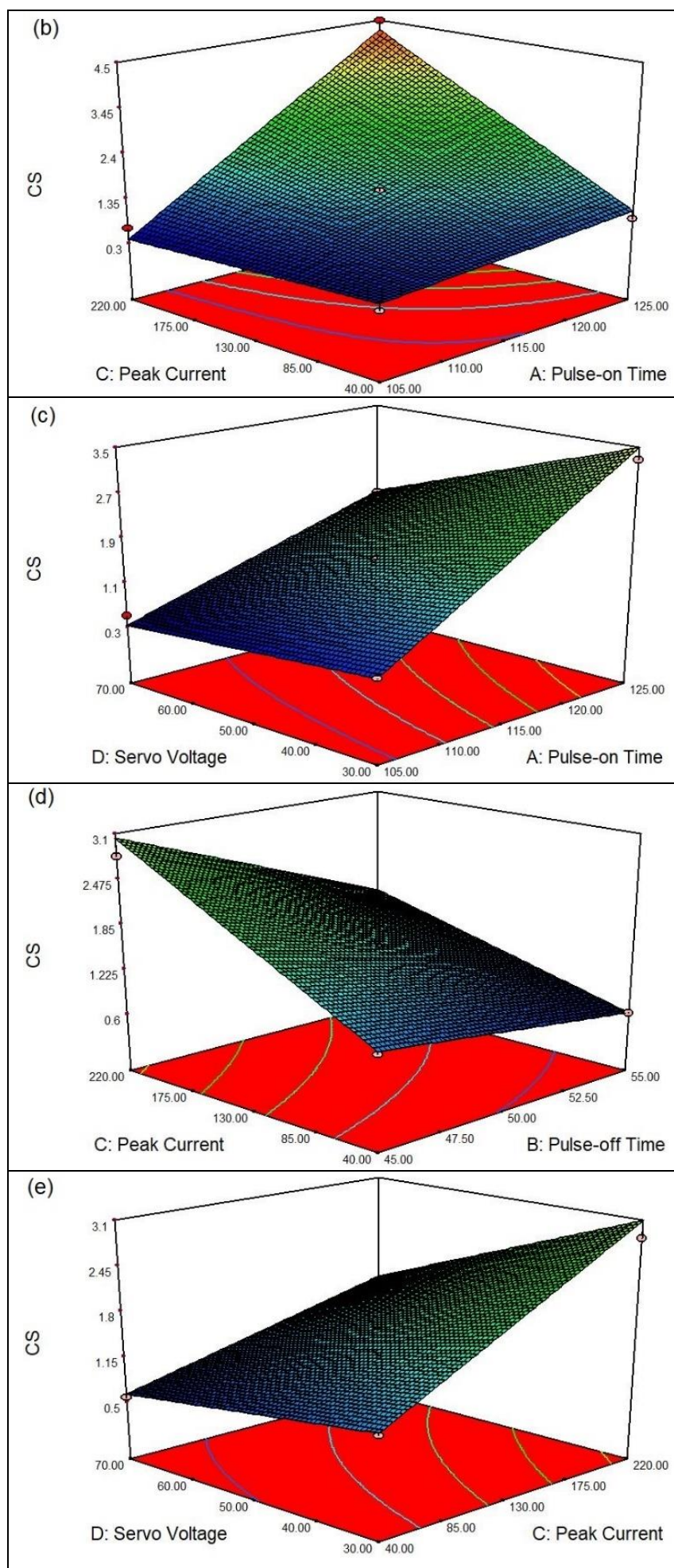

Figure 3. Interaction effects of process parameters on CS: (a) Ip-Ton vs CS; (b) Sv-T off vs CS; and (c) Ip-Sv vs CS.

Finally, optimization is carried out using to find the optimum combination of process parameters to conduct confirmation experiments. Results of confirmation experiments are presented in table 5. Moreover, the values of CS obtained in confirmation experiments are compared with the corresponding predicted values to quantify the absolute percentage of error in prediction and thus, the prediction capability of developed model is evaluated. Furthermore, at optimum setting of process parameters ( $\mathrm{T}_{\text {on }}: 124 \mu \mathrm{s}, \mathrm{T}_{\text {off: }}: 45 \mu \mathrm{s}, \mathrm{Ip}: 190 \mathrm{~A}$ and Sv: 43 Volt) provides maximum cutting speed: $4.76 \mathrm{~mm} / \mathrm{min}$.

Table 5. Confirmation experiments.

\begin{tabular}{|c|c|c|c|c|c|c|}
\hline \multicolumn{2}{|c|}{ Optimal Parameters } & \multicolumn{2}{c|}{ Cutting Speed } & $\begin{array}{c}\text { \% of } \\
\text { Error }\end{array}$ \\
\hline A & B & C & D & Predicted & Experimental & \\
\hline 121 & 47 & 200 & 33 & 4.68 & 4.55 & 0.73 \\
\hline 124 & 45 & 190 & 43 & 4.88 & 4.76 & 0.85 \\
\hline
\end{tabular}

\section{Surface integrity analysis of WEDM surfaces}

Figure 4 shows the surface appearances of the WEDMed surface of nimonic 263 at $1000 X \& 3000 X$, obtained by using FE-SEM (Make: JOEL). Three samples had been selected for microstructure observation at low CS, medium CS and high CS. From the examination of WEDMed surface, it is obvious that, molten metal is deposited in the form of lump of debris during WEDM. Also, there is a significant change in the crater and bombard size, which could be associated to the choppy distribution of discharge energy along the WEDMed surface and formation of micro-cracks are clearly visible. Figure 4 (a) shows the WEDMed surface at experimental condition corresponding to low discharge energy (LDE) which also results low cutting speed and this surface seems to be smoother as discharge craters are extremely small with the small quantity of particles, fewer microvoids are present at the surface because of LDE. Figure 4 (b) shows the surface at medium discharge energy, deep and wide craters depicted on this WEDMed surface. It is obvious from fig. 4 (c) at higher $\mathrm{T}_{\text {on }}(125 \mu \mathrm{s})$ and Ip (220 A), the discharge energy per duty cycle increases, which results in wider and deeper craters, pockmarks and lump of debris on the surface of work material. It is obvious from figure 4 (c) that the size of bombard is deeper and wider due to high $\mathrm{T}_{\text {on }}(125 \mu \mathrm{s})$ and Ip $(220 \mathrm{~A})$, which subsequently results in a high value of CS $(4.27 \mathrm{~mm} / \mathrm{min})$. High $\mathrm{T}_{\text {on }}$ value, increases the duration of spark for a longer period and high Ip value increases the pulse discharge energy on cutting zone and thus, results in higher CS.

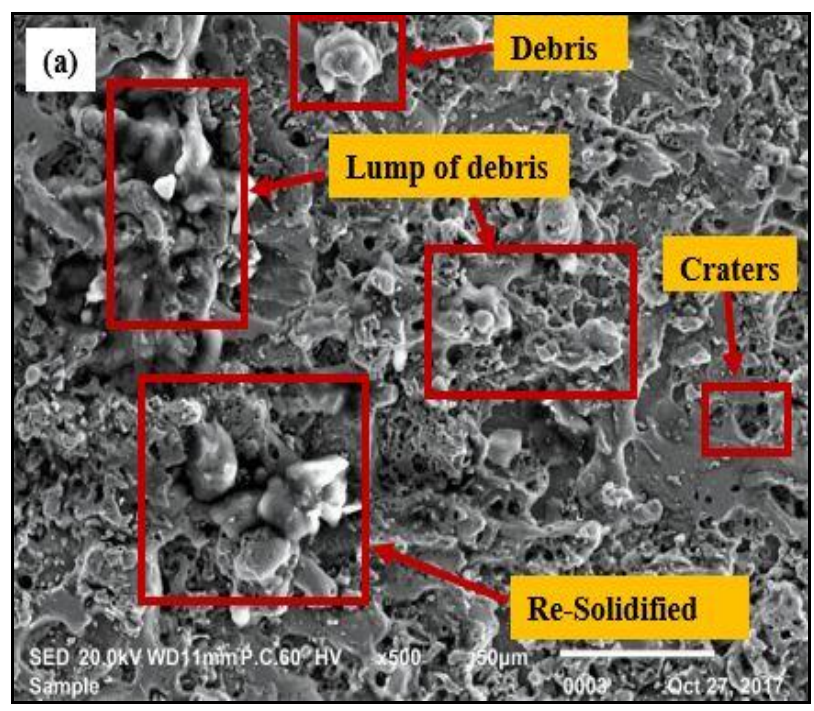



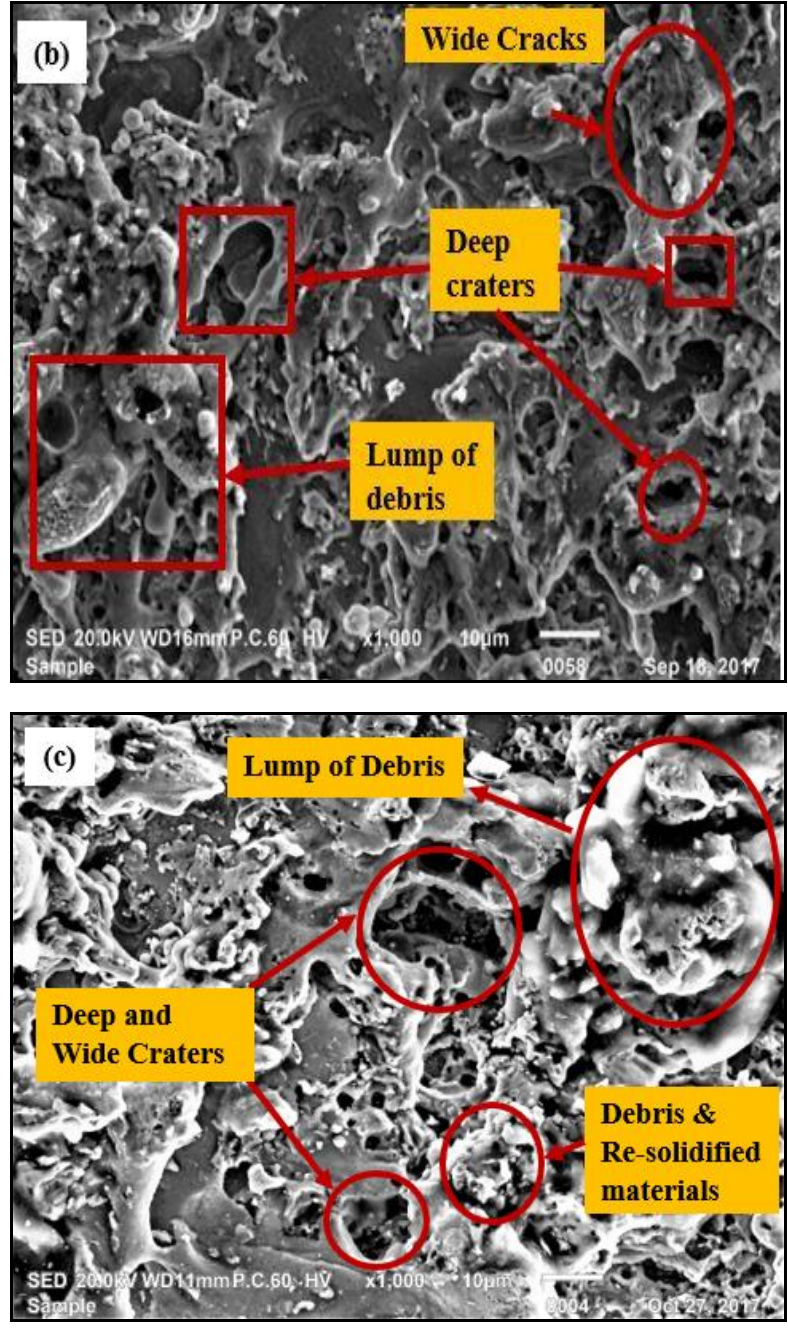

Figure 4. Microstructure of the WEDMed surfaces at (1000X \& 3000X): (a) At ex. no. 3 at LDE; (b) At ex. no. $21 \mathrm{MDE}$; and (c) At ex. no. 20 at HDE.

\section{Conclusions and future scope}

In this study a parametric study, modeling and optimization of WEDM process of Hastelloy C22 has been fulfilled based on BBD were conducted to develop empirical model of process. Following conclusion has been drawn from the analysis of the results:

- It has been observed that factor $T_{\text {on }}$ is most significant input process parameter for CS, followed by Ip. It was also noticed that $\mathrm{T}_{\text {off }}$ and $\mathrm{Sv}$ have less significant effects on responses.

- It is also observed that input parameters are having an interaction effect on cutting speed. It was also depicted CS increases with increase in Ip and $T_{\text {on }}$ and with decrease in $\mathrm{T}_{\text {off. }}$.

- Higher values of $\mathrm{T}_{\text {on }}$ and Ip leads to wire breakage. In addition, if the $\mathrm{Sv}$ is too low, an amount of discharge energy was absorbed by wire tool and debris in the gap cannot be flushed properly by the dielectric, it results in the arcing and wire breakages occur. Four experiments were not performed due to wrong parametric setting which results in wire breakage.
- Surface topography reveals that WEDMed surfaces at LDE appears to be smoother as discharge craters are extremely small with the small amount of de-bris, fewer micro-voids are present on the surface due to low discharge energy during WEDM operation.

- The developed empirical model is found best suited as it displays the very low value of prediction error $(\sim 1 \%)$ and hence, can be utilized by future users for prospective application of the technique. Pulse-on time: $124 \mu \mathrm{s}$, Pulse-off time: $45 \mu$ s, Peak current: 190 Amp and Servo voltage: 43 Volt, are found optimum for the maximized value of CS.

- Future study could consider the tribological and microstructure study of WEDM machined miniature parts: gear, spline etc. for obtaining better insight of the process.

\section{References}

1. E. Jameson, Electrical discharge machining, (2001)

2. B. Fleming, The EDM how-to book, (2005).

3. K. H. Ho, S. T. Newman, S. Rahimifard, and R. D. Allen, State of the art in wire electrical discharge machining (WEDM), Int. J. Mach. Tools Manuf., 44, no. 12-13, 1247-1259, (2004).

4. B. Singh and J. P. Misra, A Critical Review of Wire Electric Discharge Machining, Ann. DAAAM Proc. Int. DAAAM Symp., 249-266, (2016).

5. N. Tosun, C. Cogun, and A. Inan, The Effect of Cutting Parameters on Workpiece Surface Roughness in Wire EDM, Mach. Sci. Technol., 7, no. 2, 209-219, (2003).

6. T. Singh, J. P. Misra, and B. Singh, Experimental Investigation of Influence of Process Parameters on MRR during WEDM of Al6063 alloy, Materials Today: Proceedings, 4, 2242-2247, (2017).

7. A. Kumar, V. Kumar, and J. Kumar, Parametric effect on Wire-breakage frequency and surface topography in WEDM of pure titanium, J. Mech. Eng. Technol., 1, 51-56, 2013.

8. P. Shandilya, P. K. Jain, and J. P. Misra, Experimental investigation during wire electric discharge cutting of $\mathrm{SiCp} / 6061$ aluminum metal matrix composite, Ann. DAAAM Proc. Int. DAAAM Symp., 21, 1091-1092, 2010.

9. N. Tosun, C. Cogun, and G. Tosun, "A study on kerf and material removal rate in wire electrical discharge machining based on Taguchi method," J. Mater. Process. Technol., 152, 316-322, 2004.

10. R. Chalisgaonkar and J. Kumar, Multi-response optimization and modeling of trim cut WEDM operation of commercially pure titanium (CPTi) considering multiple user's preferences, Eng. Sci. Technol. an Int. J., 18, pp. 125-134, 2014.

11. R. H. Myers, D. C. Montgomery, and C. M. Anderson-cook, Response Surface Methodology, Wiley, 1-1247, 2009. 